\title{
Primary Hyperparathyroidism with Severe Hypercalcemia During Pregnancy
}

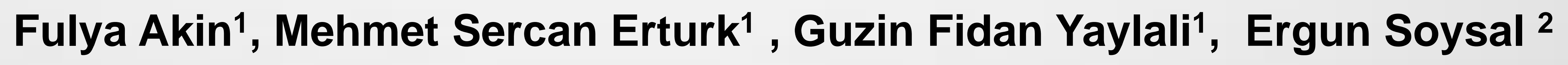 \\ ${ }^{1}$ Pamukkale University, Faculty of Medicine, Department of Endocrinology and Metabolism, \\ Denizli, 20070, Turkey \\ 2Pamukkale University, Faculty of Medicine, Department of Internal Medicine, Denizli, 20070, Turkey
}

\section{Introduction}

Primary hyperparathyroidism (PHPT) commonly goes unrecognized due to the physiological changes of pregnancy. Hypoalbuminemia, calcium transport across the placenta, and an increased glomerular filtration rate all contribute to the appearance of lower calcium levels in the pregnant patient. In addition, estrogen is thought to inhibit parathyroid hormone (PTH)-mediated bone resorption, causing a dose-related reduction in serum calcium in pregnancy.PHPT is rarely diagnosed during pregnancy but is associated with significant maternal and fetal morbidity and mortality. Gestational age and the severity of hypercalcemia should be taken into account when assessing the riskbenefit balance of a conservative approach versus parathyroid surgery.Subcutaneous calcitonin is also utilized and has a category $C$ rating. Current evidence supports parathyroidectomy is the main treatment, performed preferably during the second trimester, when the serum calcium is above $11 \mathrm{mg} / \mathrm{dL}$. We report the clinical course of a woman with newly diagnosed gestational PHPT who refused minimally invasive parathyroidectomy during and after pregnancy.

\section{Case report}

A 23 -year-old primigravida woman presented during her 35th week of gestation with a two day history of severe nausea and vomiting.She denied any history of abdominal pain, constipation, polydipsia, polyuria, weight loss, muscle weakness. She had no history of calcium disorders, kidney stones, fractures, osteoporosis. She was not taking any drugs which could influence her calcium status. Admission laboratory tests revealed several hypercalcemia with a adjusted calcium level 18.43 $\mathrm{mg} / \mathrm{dL}$, phosphorus $2.39 \mathrm{mgldl}$ and parathyroid hormone(PTH) $775 \mathrm{pg} / \mathrm{ml}$ serum alkaline phosphatase(ALP) 157ı/L urinary calcium level $503 \mathrm{mg} / 24 \mathrm{hr}$. $\mathrm{hr}$ Other blood electrolytes, including magnesium and potassium ,1,25-dihydroxycholecalciferol, ECG, and corrected QT intervals were all within normal limits .Ultrasound examination of her neck identified one suspicious parathyroid enlargement at the inferior pole of thyroid gland measuring $17 \times 13 \times 11 \mathrm{~mm}$. Severe hypercalcemia was treated with intravenous saline infusion, calcitonin,diuretic and corticosteroids. She denied minimally invasive parathyroidectomy during third trimester. When her serum calcium level dropped the level of 13.5 mgldl, cesaran section was performed. The baby was healthy and normocalcemic with a calcium level of $9.8 \mathrm{mg} / \mathrm{dL}$ and a PTH level of $121 \mathrm{pg} / \mathrm{mL}$ on day 1 . After pregnancy she again refused parathyroidectomy and medical treatment and the mother and baby were discharged home on the six day after delivery.

\section{Discussion}

Hypercalcemia during pregnancy is uncommon and mostly associated with PHPT. Because of up to $80 \%$ gravid patients with PHPT are asymptomatic, diagnosis is more difficult. Complications associated with PHP in pregnancy have been reported to occur in up to $67 \%$ of mothers and $80 \%$ of fetuses. Hyperemesis gravidarum, preeclampsia, urinary tract infections, muscle weakness, mental status changes can be seen
There are elevated miscarriage rate.Reported fetal complications include intrauterine growth retardation, preterm delivery, intrauterin fetal demise, postpartum neonatal tetany. Hypercalcemia during the pregnancy is a life threatening condition for both mother and fetus. If asymptomatic moderate hypercalcemia $(<12 \mathrm{mg} / \mathrm{dl})$ is present, it is appropriate to follow a conservative policy. Oral hydration and decrement of dietary calcium intake with are recommended. The patient can be followed up to the end of first trimester as long as calcium level is kept below $12 \mathrm{mg} / \mathrm{dl}$ (1). Beside adequate hydration and diuresis, calcitonin is the other medical treatment options. Emergent parathyroidectomy should be considered if the clinical stabilization and control of hypercalcemia can not be accomplished. Parathyroidectomy is recommended during the second trimester when the risk of anesthesia-induced preterm delivery is lowest and organogenesis is complete $(2,3)$. The minimally invasive surgery or bilateral neck exploration can be preferred $(4,5)$. Surgery during the third trimester classically has a higher risk of preterm labor. Carella and Gossain reviewed 38 women who underwent parathyrodectomy 7 women during first trimester, 18 during second, and 12 during third trimester. A 58\% perinatal complication rate was reported after third trimester parathyroidectomy (3). At the same time cases of uncomplicated third trimester have been reported (6). In our case at presentation blood calcium level is greater then $18 \mathrm{mg} / \mathrm{dl}$. She denied minimally invasive parathyroidectomy during third trimester. When her serum calcium level dropped the level of $13.5 \mathrm{mgldl}$ with medication, caesarean section was performed. The baby was healthy and normocalcemic with a calcium level of $9.8 \mathrm{mg} / \mathrm{dL}$ and a PTH level of $121 \mathrm{pg} / \mathrm{mL}$ on day 1 .

\section{Conclusions}

Timely recognition and effective management of PHP in pregnancy is important because it represents a preventable cause of fetal and maternal morbidity and mortality. Although our case lately presented, both maternal and fetal outcomes was excellent with medical intervention.

\section{References}

1.Cunnigham FG, MacDonald PC, Gant Nf et al. Parathyroid disease. Williams Obstetrics (ed). Edited by Stamford CT; Appleton\&Lange; 1997:1230-1.

2. Van Dalen A, Smith CP, van Vroonhoven TJ. Minimally invasive surgery for solitary parathyroid adenomas in patients with primary hyperparathyroidism: Role of US with supplemental CT Radiology 2001;220:631-9.

3. Carella MJ, Gossain VV. Hyperparathyroism and pregnancy: case report and review. J Gen intern Med 1992;7:448-53

4. Bilezikian JP, Khan AA, Potts JT Jr; Third International Workshop on the Management of Asymptomatic Primary Hyperthyroidism. Guidelines fort he management of asymptomatic primary hyperparathyroidism: Summary statement from the third international workshop. J Clin Endocrinol Metab 2009;94:335-9.

5. Bilezikian, JP, Potts, JT, El-Hajj Fuleihan, et al. Summary statement from a workshop on asymptomatic primary hyperparathyroidism: a perspective for the 21 st century. J Bone Min Res 2002;17:2-11.

6. Schnatz P, Thaxton S. Parathyroidectomy in the third trimester of pregnancy. Obstet Gynecol Surv 2005;60:672-82 